\title{
A CHARACTERIZATION OF THE INTERSECTION OF $L^{1}$-SPACES
}

\section{SIGURĐUR HELGASON}

Let $\mathfrak{C}(S)$ denote the space of real valued continuous functions vanishing at infinity on a locally compact Hausdorff space $S$. If $\mu$ is a positive Radon measure on $S$ and $p \geqq 1$, the space $A=L^{p}(\mu) \cap \mathbb{C}(S)$ is a Banach space under the norm $\|x\|=\left[\int_{S}|x(t)|^{p} d \mu\right]^{1 / p}+\|x\|_{\infty}$; here $L^{p}$ denotes the space of real $p^{\text {th }}$ power integrable functions on $S$ (no identification modulo measure zero!), and $\|x\|_{\infty}$ denotes $\sup _{t \in S}|x(t)|$. Further, if $\mu_{i}(i \in I)$ is a family of positive Radon measures on $S$ the space $A=\bigcap_{i} L^{1}\left(\mu_{i}\right) \cap \mathbb{S}(S)$ is a locally convex topological vector space in which a fundamental system of neighborhoods of 0 is given by all finite intersections of sets of the form

$$
V=\left\{x\left|\int_{S}\right| x(t) \mid d \mu_{i}+\|x\|_{\infty}<\varepsilon\right\}
$$

where $i$ varies through $I$ and $\varepsilon$ varies through all positive real numbers. The spaces are ordered in the natural way, the relation $x \leqq y$ meaning $x(t) \leqq y(t)$ for all $t \in S$. We write $|x|$ for the function $t \rightarrow|x(t)|$. The neighborhoods $V$ above have the property that the relations $y \in V$ and $|x| \leqq|y|$ imply $x \in V$. Neighborhoods of 0 with this property in an ordered topological vector space will be called full neighborhoods of 0 . We also recall that a subset $M$ of a topological vector space is called bounded if for every neighborhood $U$ of 0 there exists a real number $\lambda>0$ such that $M \subset \lambda U$.

The spaces $\boldsymbol{A}$ are furthermore topological algebras under usual pointwise addition and multiplication and satisfy the following conditions:

(i) $A$ is an ideal in $\subseteq(S)$.

(ii) The functions in $A$ have no common zero.

(iii) $A$ has a fundamental system of full neighborhoods of 0 .

(iv) If $M$ is a bounded subset of $A$ and $g$ is in the uniform closure of $M$, then $g \in A$.

(i), (ii), and (iii) are obvious and (iv) follows from Fatou's lemma.

Received November 9, 1955.

Work done in connection with the Air Force Analysis Research Project at the University of Chicago 1955. 
Our purpose is now to show the converse of the statements above, namely the following

TheORem. Let $A$ be a locally convex topological algebra such that $A$ is a subalgebra of $\mathfrak{S}(S)$ and the conditions (i)-(iv) are satisfied. Then there exists a family of positive Radon measures $\mu_{k}$ on $S$ such that

$$
A=\cap_{k} L^{1}\left(\mu_{k}\right) \cap \mathfrak{C}(S) \text {. }
$$

Proof. Let $x \in A$ and consider the mapping $y \rightarrow x y$ from $\mathbb{E}(S)$ to $A$. It follows directly from (iii) that this mapping is continuous $(\mathbb{E}(S)$ of course being equipped with the uniform topology). Therefore, if $F$ is a continuous linear functional on $A$, the mapping $y \rightarrow F(x y)$ is a continuous linear functional on $\mathfrak{E}(S)$. Hence we have

$$
F(x y)=\int_{S} y(t) d \mu_{x}(t) \quad \text { for all } y \in \mathfrak{C}(S)
$$

where $\mu_{x}$ is a uniquely determined bounded Radon measure on $S$. If $x, y, z \in A$ we have

$$
F(x y z)=\int_{S} y(t) x(t) d \mu_{z}(t)=\int_{S} y(t) z(t) d \mu_{x}(t)
$$

and by the uniqueness

$$
x \mu_{z}=z \mu_{x} .
$$

On the open subset $S_{x}=\{t \mid x(t) \neq 0\}$ we consider the measure

$$
\mu_{F}^{x}(A)=\int_{A} \frac{1}{x(t)} d \mu_{x}(t)
$$

defined at least for the bounded Borel subsets of $S_{x}$. From (1) it follows, that $\mu_{F}^{x}$ and $\mu_{F}^{z}$ are identical on the intersection $S_{x} \cap S_{z}$ and since by condition (ii) the sets $S_{x}$ cover $S$ it is not hard to show that there exists a unique Radon measure $\mu_{F}$ on $S$ such that for each $x \in A, \mu_{F}{ }^{x}$ is identical to the restriction of $\mu_{F}$ to $S$. (See e.g. [1, p. 68].) Hence we obtain for $x \in A$

$$
F\left(x^{2}\right)=\int_{S} x d \mu_{x}=\int_{S_{x}} x d \mu_{x}=\int_{S_{x}} x^{2} d \mu_{F}=\int_{S} x^{2} d \mu_{F},
$$

the third equality being a consequence of the equation

$$
\int_{S_{x}} f(t) d \mu_{x}=\int_{S_{x}} f(t) x(t) d \mu_{F}
$$


which holds for all continuous functions $f(t)$ that vanish outside a compact subset of $S_{x}$. It follows that

$$
F(x y)=\int_{S} x(t) y(t) d \mu_{F}(t) \quad \text { for all } x, y \in A .
$$

From the conditions (i) and (ii) it is easy to conclude, that $A$ contains all continuous functions on $S$ of compact support. If $x(t)$ is such a function we obtain from (2) the relation

$$
F(x)=\int_{S} x(t) d \mu_{F}(t) .
$$

The measures $\mu_{F}$ and the components $\mu_{F}{ }^{+}$and $\mu_{F^{-}}$in the Jordan decomposition $\mu_{F}=\mu_{F}{ }^{+}-\mu_{F^{-}}$are in general unbounded. However, all the functions $x(t)$ are integrable with respect to them. We omit the proof of this statement since it can be carried through in exactly the same way as the proof of the similar statement in lemma 1 of [2].

To complete the proof of the theorem we shall now show conversely that every function $g \in \mathbb{C}(S)$ which is integrable with respect to all the measures $\mu_{F}{ }^{+}+\mu_{F^{-}}$belongs to $A$. To do this let $x_{n}$ be a sequence of continuous functions of compact support such that $x_{n}(t)$ converges to $g(t)$ uniformly on $S$ and such that $\left|x_{n}(t)\right| \leqq|g(t)|$ for every $t$ and every $n$. As remarked before, all $x_{n}$ belong to $A$ and using Lebesgue's dominated convergence theorem we conclude from (3) that $F\left(x_{n}\right)$ is a convergent sequence for every continuous linear functional $F$. In particular, the sequence $\left(x_{n}\right)$ is weakly bounded. In a locally convex topological vector space it is known (see [3, Theorem 7]) that a weakly bounded set is bounded, so property (iv) implies $g \in A$ as desired.

The question arises whether the topology of $A$ is necessarily the same as the "natural" topology on $\bigcap_{k} L^{1}\left(\mu_{k}\right) \cap \mathbb{C}(S)$, that is the topology described in the beginning of this article. The answer is negative, even in simple cases. For example, if $L^{1}$ is the space of Lebesgue integrable functions on $R$, the algebra $A=L^{1} \cap \mathbb{E}(R)$ can be given two distinct topologies both of which satisfy the conditions (i)-(iv). These topologies are obtained by means of the norms

$$
p(x)=\int_{-\infty}^{\infty}|x(t)| d t \quad \text { and } \quad q(x)=\int_{-\infty}^{\infty}|x(t)| d t+\|x\|_{\infty}
$$

respectively. 


\section{REFERENCES}

1. N. Bourbaki, Intégration (Actualités Sci. Ind. 1175), Paris, 1952.

2. S. Helgason, Multipliers of Banach algebras, to appear in the Annals of Mathematics.

3. G. W. Mackey, On convex topological linear spaces, Trans. Amer. Math. Soc. 60 (1946), 519-537.

MASSACHUSETTS INSTITUTE OF TECHNOLOGY, CAMBRIDGE, MASS., U.S.A. 